Algebraic $8 \mathcal{G}$ Geometric $\mathcal{T}_{\text {opology }}$

Volume 5 (2005) 369-378

Published: 30 April 2005

ATG

\title{
All integral slopes can be Seifert fibered slopes for hyperbolic knots
}

\author{
Kiminiko Motegi \\ HyUn-Jong SONG
}

\begin{abstract}
Which slopes can or cannot appear as Seifert fibered slopes for hyperbolic knots in the 3 -sphere $S^{3}$ ? It is conjectured that if $r$-surgery on a hyperbolic knot in $S^{3}$ yields a Seifert fiber space, then $r$ is an integer. We show that for each integer $n \in \mathbb{Z}$, there exists a tunnel number one, hyperbolic knot $K_{n}$ in $S^{3}$ such that $n$-surgery on $K_{n}$ produces a small Seifert fiber space.
\end{abstract}

AMS Classification 57M25, 57M50

Keywords Dehn surgery, hyperbolic knot, Seifert fiber space, surgery slopes

This paper is dedicated to Donald M. Davis on the occasion of his 60th birthday.

\section{Introduction}

Let $K$ be a knot in the 3 -sphere $S^{3}$ with a tubular neighborhood $N(K)$. Then the set of slopes for $K$ (i.e., $\partial N(K)$-isotopy classes of simple loops on $\partial N(K)$ ) is identified with $\mathbb{Q} \cup\{\infty\}$ using preferred meridian-longitude pair so that a meridian corresponds to $\infty$. A slope $\gamma$ is said to be integral if a representative of $\gamma$ intersects a meridian exactly once, in other words, $\gamma$ corresponds to an integer under the above identification. In the following, we denote by $(K ; \gamma)$ the 3-manifold obtained from $S^{3}$ by Dehn surgery on a knot $K$ with slope $\gamma$, i.e., by attaching a solid torus to $S^{3}-\operatorname{int} N(K)$ in such a way that $\gamma$ bounds a meridian disk of the filled solid torus. If $\gamma$ corresponds to $r \in \mathbb{Q} \cup\{\infty\}$, then we identify $\gamma$ and $r$ and write $(K ; r)$ for $(K ; \gamma)$.

We denote by $\mathcal{L}$ the set of lens slopes $\left\{r \in \mathbb{Q} \mid \exists\right.$ hyperbolic knot $K \subset S^{3}$ such that $(K ; r)$ is a lens space $\}$, where $S^{3}$ and $S^{2} \times S^{1}$ are also considered as lens spaces. Then the cyclic surgery theorem [7] implies that $\mathcal{L} \subset \mathbb{Z}$. A 
result of Gabai [10, Corollary 8.3] shows that $0 \notin \mathcal{L}$, a result of Gordon and Luecke [14] shows that $\pm 1 \notin \mathcal{L}$. In [19] Kronheimer and Mrowka prove that $\pm 2 \notin \mathcal{L}$. Furthermore, a result of Kronheimer, Mrowka, Ozsváth and Szabó [20] implies that $\pm 3, \pm 4 \notin \mathcal{L}$. Besides, Berge [4, Table of Lens Spaces] suggests that if $n \in \mathcal{L}$, then $|n| \geq 18$ and not every integer $n$ with $|n| \geq 18$ appears in $\mathcal{L}$. Fintushel and Stern [9] had shown that 18-surgery on the $(-2,3,7)$ pretzel knot yields a lens space.

Which slope (rational number) can or cannot appear in the set of Seifert fibered slopes $\mathcal{S}=\left\{r \in \mathbb{Q} \mid \exists\right.$ hyperbolic knot $K \subset S^{3}$ such that $(K ; r)$ is Seifert fibered $\}$ ? It is conjectured that $\mathcal{S} \subset \mathbb{Z}[12$.

The purpose of this paper is to prove:

Theorem 1.1 For each integer $n \in \mathbb{Z}$, there exists a tunnel number one, hyperbolic knot $K_{n}$ in $S^{3}$ such that $\left(K_{n} ; n\right)$ is a small Seifert fiber space (i.e., a Seifert fiber space over $S^{2}$ with exactly three exceptional fibers).

Remark Since $K_{n}$ has tunnel number one, it is embedded in a genus two Heegaard surface of $S^{3}$ and strongly invertible [26, Lemma 5]. See [22, Question 3.1].

Theorem 1.1. together with the previous known results, shows:

Corollary $1.2 \quad \mathcal{L} \varsubsetneqq \mathbb{Z} \subset \mathcal{S}$.

\section{Remarks}

(1) For the set of reducing slopes $\mathcal{R}=\left\{r \in \mathbb{Q} \mid \exists\right.$ hyperbolic knot $K \subset S^{3}$ such that $(K ; r)$ is reducible $\}$, Gordon and Luecke [13] have shown that $\mathcal{R} \subset \mathbb{Z}$. In fact, the cabling conjecture [1] asserts that $\mathcal{R}=\emptyset$.

(2) For the set of toroidal slopes $\mathcal{T}=\left\{r \in \mathbb{Q} \mid \exists\right.$ hyperbolic knot $K \subset S^{3}$ such that $(K ; r)$ is toroidal $\}$, Gordon and Luecke [15] have shown that $\mathcal{T} \subset \mathbb{Z} / 2$ (integers or half integers). In 28], Teragaito shows that $\mathbb{Z} \subset \mathcal{T}$ and conjectures that $\mathcal{T} \varsubsetneqq \mathbb{Z} / 2$.

Acknowledgements We would like to thank the referee for careful reading and useful comments.

The first author was partially supported by Grant-in-Aid for Scientific Research (No. 15540095), The Ministry of Education, Culture, Sports, Science and Technology, Japan. 


\section{Hyperbolic knots with Seifert fibered surgeries}

Our construction is based on an example of a longitudinal Seifert fibered surgery given in [17.

Let $k \cup c$ be a 2-bridge link given in Figure 1 and let $K_{n}$ be a knot obtained from $k$ by $\frac{1}{-n+4}$-surgery along $c$.

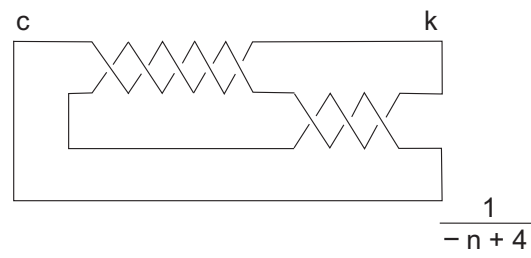

Figure 1: $K_{n}$

We shall say that a Seifert fiber space is of type $S^{2}\left(n_{1}, n_{2}, n_{3}\right)$ if it has a Seifert fibration over $S^{2}$ with three exceptional fibers of indices $n_{1}, n_{2}$ and $n_{3}\left(n_{i} \geq 2\right)$. Since $K_{4}$ is unknotted, $\left(K_{4} ; 4\right)$ is a lens space $L(4,1)$. For the other $n$ 's, we have:

Lemma $2.1\left(K_{n} ; n\right)$ is a small Seifert fiber space of type $S^{2}(3,5,|4 n-15|)$ for any integer $n \neq 4$.

Proof Since the linking number of $k$ and $c$ is one (with suitable orientations), $\left(K_{n} ; n\right)$ has surgery descriptions as in Figure 2 .

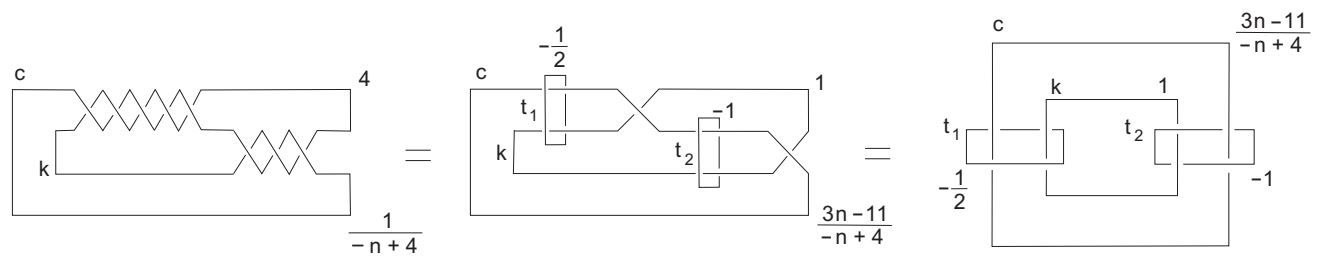

Figure 2: Surgery descriptions of $\left(K_{n} ; n\right)$

Let us take the quotient by the strong inversion of $S^{3}$ with an axis $L$ as shown in Figure 3 ,

Then we obtain a branch knot $b^{\prime}$ which is the image of the axis $L$. The Montesinos trick $([25], 6])$ shows that $-\frac{1}{2},-1, \frac{3 n-11}{-n+4}$ and 1 -surgery on $t_{1}, t_{2}, c$ and 

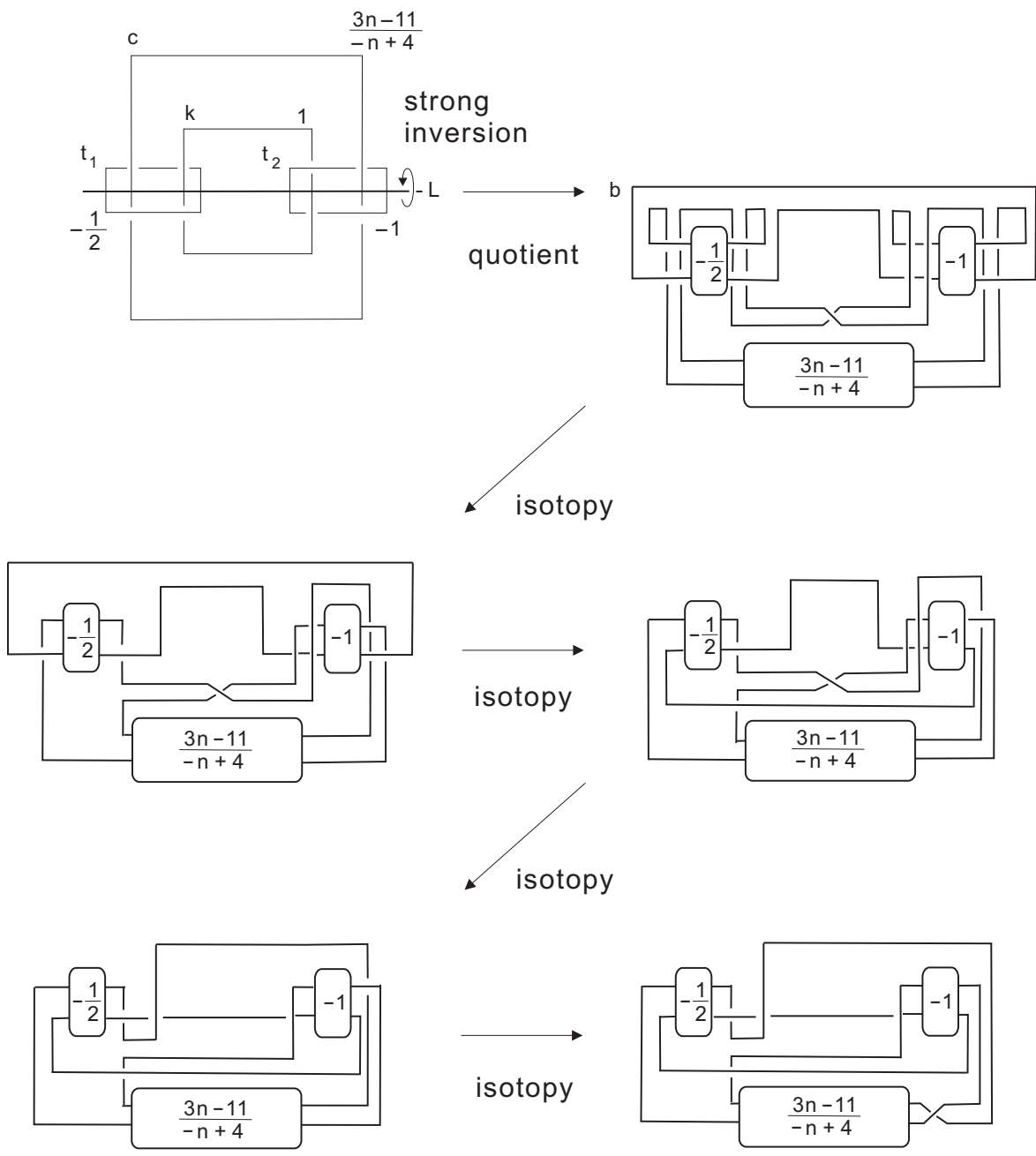

Figure 3

$k$ in the upstairs correspond to $-\frac{1}{2},-1, \frac{3 n-11}{-n+4}$ and 1-untangle surgery on $b^{\prime}$ in the downstairs, where an $r$-untangle surgery is a replacement of $\frac{1}{0}$-untangle by $r$-untangle. (We adopt Bleiler's convention [5] on the parametrization of rational tangles.) These untangle surgeries convert $b^{\prime}$ into a link $b$ (Figure 3).

Following the sequence of isotopies in Figures 3 and 4, we obtain a Montesinos link $M\left(\frac{2}{5},-\frac{2}{3}, \frac{n-4}{4 n-15}\right)$.

Since $\left(K_{n} ; n\right)$ is the double branched cover of $S^{3}$ branched over the Montesinos 

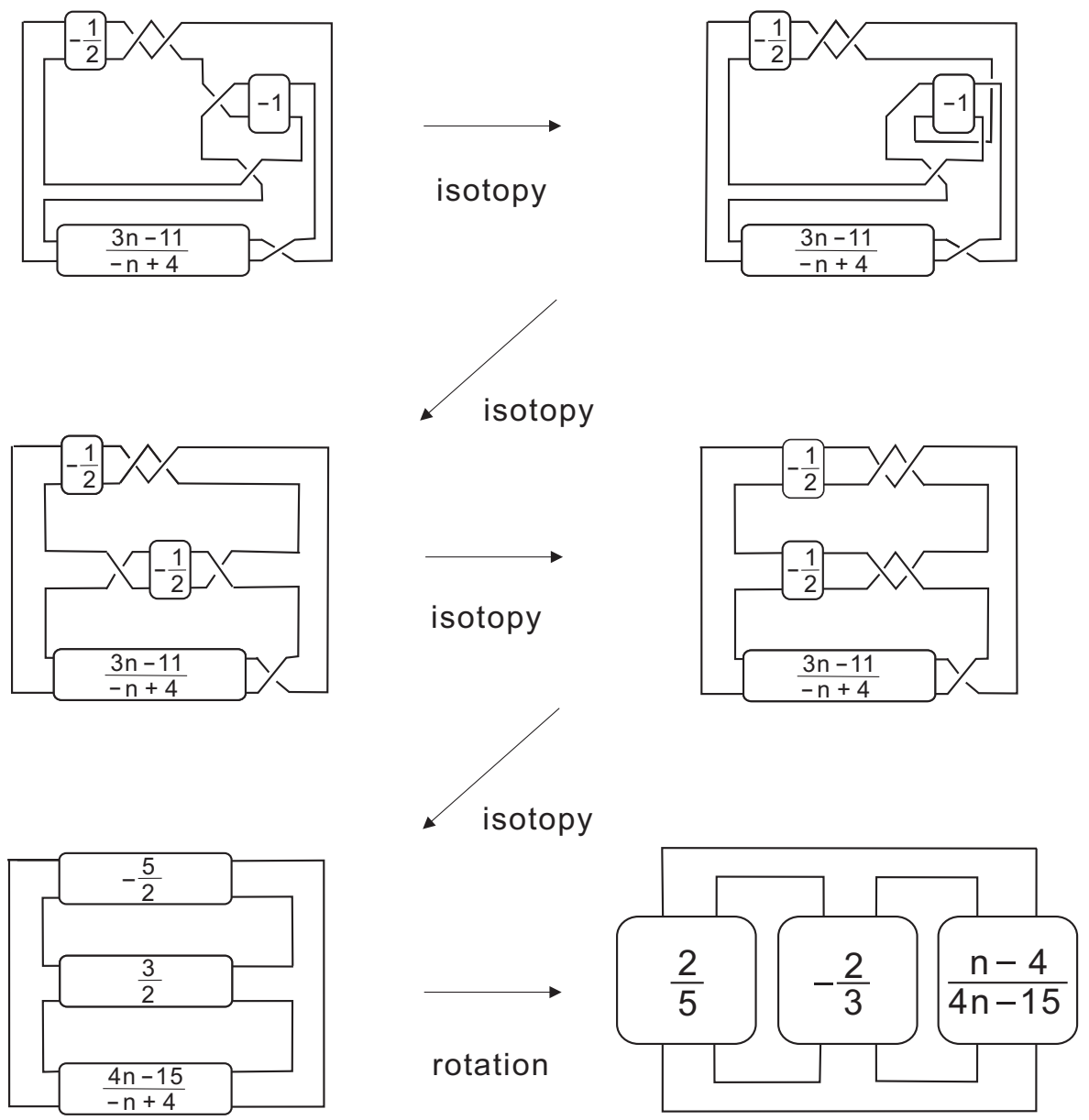

Figure 4: Continued from Figure 3

link $M\left(\frac{2}{5},-\frac{2}{3}, \frac{n-4}{4 n-15}\right),\left(K_{n} ; n\right)$ is a Seifert fiber space of type $S^{2}(3,5,|4 n-15|)$ as desired.

Lemma 2.2 The knot $K_{n}$ is hyperbolic if $n \neq 3,4,5$.

Proof Note that the 2-bridge link given in Figure 1 is not a $(2, p)$-torus link, and hence by [23] it is a hyperbolic link. If $n \neq 3,4,5$, then $|-n+4|>1$ and it follows from [1, Theorem 1] (also [3. Theorem 1.2]) that $K_{n}$ is a hyperbolic knot. See also [16, Corollary A.2], 24, Theorem 1.2] and [2, Theorem 1.1]. 
Remark It follows from 21, 18, that $K_{n}$ is a nontrivial knot except when $n=4$. An experiment using Weeks' computer program "SnapPea" 31] suggests that $K_{3}$ and $K_{5}$ are hyperbolic, but we will not use this experimental results.

Lemma 2.3 The knot $K_{n}$ has tunnel number one for any integer $n \neq 4$.

Proof Since the link $k \cup c$ is a two-bridge link, the tunnel number of $k \cup c$ is one with unknotting tunnel $\tau$; A regular neighborhood $N(k \cup c \cup \tau)$ is a genus two handlebody and $S^{3}-\operatorname{int} N(k \cup c \cup \tau)$ is also a genus two handlebody, see Figure [5]

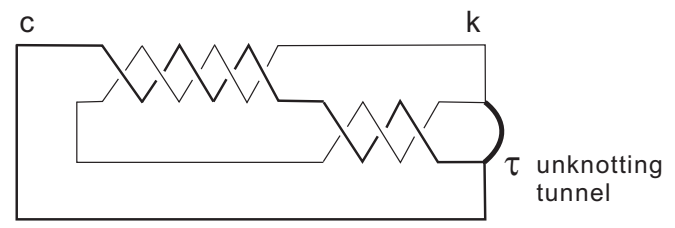

Figure 5

Then the general fact below (in which $k \cup c$ is not necessarily a two-bridge link) shows that the tunnel number of $K_{n}$ is less than or equal to one. Since our knot $K_{n}(n \neq 4)$ is knotted in $S^{3}$, the tunnel number of $K_{n}$ is one.

Claim 2.4 Let $k \cup c$ be a two component link in $S^{3}$ which has tunnel number one. Assume that $c$ is unknotted in $S^{3}$. Then every knot obtained from $k$ by twisting along $c$ has tunnel number at most one.

Proof Let $\tau$ be an unknotting tunnel and $V$ a regular neighborhood of $k \cup c \cup \tau$ in $S^{3} ; V$ is a genus two handlebody. Since $\tau$ is an unknotting tunnel for $k \cup c$, by definition, $W=S^{3}-\operatorname{int} V$ is also a genus two handlebody. Take a small tubular neighborhood $N(c) \subset \operatorname{int} V$ and perform $-\frac{1}{n}$-surgery on $c$ using $N(c)$. Then we obtain a knot $k_{n}$ as the image of $k$ and obtain a genus two handlebody $V\left(c ;-\frac{1}{n}\right)$. Note that $V\left(c ;-\frac{1}{n}\right)$ and $W$ define a genus two Heegaard splitting of $S^{3}$, see Figure [6] where $c_{n}^{*}$ denotes the core of the filled solid torus.

Then it is easy to see that an arc $\tau_{n}$ given by Figure [ is an unknotting tunnel for $k_{n}$ as desired.

Now we are ready to prove Theorem 1.1. Lemmas 2.1, 2.2 and 2.3 show that our knots $K_{n}$ enjoy the required properties, except for $n=3,4,5$. To prove 


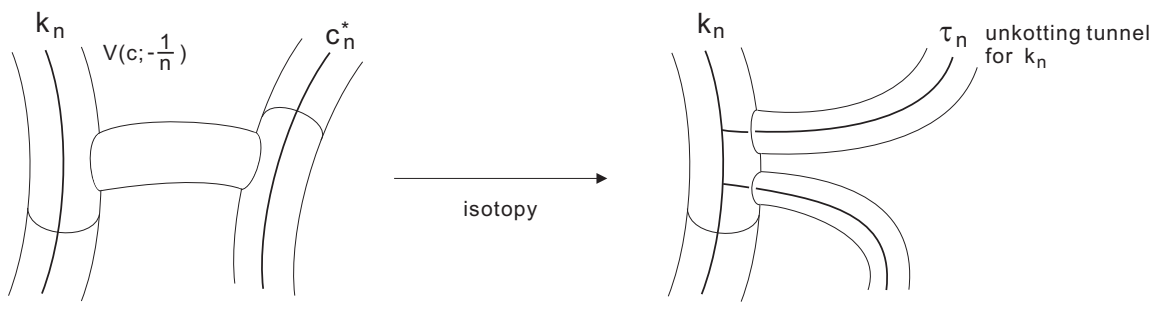

Figure 6

Theorem 1.1 we find hyperbolic knots $K_{n}^{\prime}$ so that $\left(K_{n}^{\prime} ; n\right)$ is Seifert fibered for $n=3,4,5$ (instead of showing that $K_{3}, K_{5}$ are hyperbolic). As the simplest way, let $K_{3}^{\prime}, K_{4}^{\prime}$ and $K_{5}^{\prime}$ be the mirror image of $K_{-3}, K_{-4}$ and $K_{-5}$, respectively. Since $K_{-3}, K_{-4}$ and $K_{-5}$ are tunnel number one, hyperbolic knots by Lemmas 2.2 and 2.3. their mirror images $K_{3}^{\prime}, K_{4}^{\prime}$ and $K_{5}^{\prime}$ are also tunnel number one, hyperbolic knots. It is easy to observe that $\left(K_{3}^{\prime} ; 3\right)\left(\operatorname{resp} .\left(K_{4}^{\prime} ; 4\right)\right.$, $\left.\left(K_{5}^{\prime} ; 5\right)\right)$ is the mirror image of $\left(K_{-3} ;-3\right)$ (resp. $\left.\left(K_{-4} ;-4\right),\left(K_{-5} ;-5\right)\right)$. By Lemma 2.1 $\left(K_{-3} ;-3\right),\left(K_{-4} ;-4\right)$ and $\left(K_{-5} ;-5\right)$ are Seifert fibered, and hence $\left(K_{3}^{\prime} ; 3\right),\left(K_{4}^{\prime} ; 4\right)$ and $\left(K_{5}^{\prime} ; 5\right)$ are also Seifert fibered. Putting $K_{n}$ as $K_{n}^{\prime}$ for $n=3,4,5$, we finish a proof of Theorem 1.1

\section{$3 \quad$ Identifying exceptional fibers}

In 24], Miyazaki and Motegi conjectured that if $K$ admits a Seifert fibered surgery, then there is a trivial knot $c \subset S^{3}$ disjoint from $K$ which becomes a Seifert fiber in the resulting Seifert fiber space, and verified the conjecture for several Seifert fibered surgeries [24, Section 6], see also [8]. Furthermore, computer experiments via "SnapPea" 31] suggest that such a knot $c$ is realized by a short closed geodesic in the hyperbolic manifold $S^{3}-K$, for details see [24. Section 9], 27.

In this section, we verify the conjecture for Seifert fibered surgeries given in Theorem 1.1

Recall that $K_{n}$ is obtained from $k$ by $\frac{1}{-n+4}$-surgery on the trivial knot $c$ (i.e., $(n-4)$-twist along $c)$, see Figure 1. Denote by $c_{n}$ the core of the filled solid torus. Then $K_{n} \cup c_{n}$ is a link in $S^{3}$ such that $c_{n}$ is a trivial knot.

Lemma 3.1 After $n$-surgery on $K_{n}, c_{n}$ becomes an exceptional fiber of index $|4 n-15|$ in the resulting Seifert fiber space $\left(K_{n} ; n\right)$. 
Proof Following the sequences given by Figures 3 and 4 , we have a Montesinos link with three $\operatorname{arcs} \gamma, \tau_{1}$ and $\tau_{2}$ as in Figure [7 where $n=1$ in the final Montesinos link, and $\gamma, \tau_{1}, \tau_{2}$ and $\kappa$ are the images of $c, t_{1}, t_{2}$ and $k$, respectively.
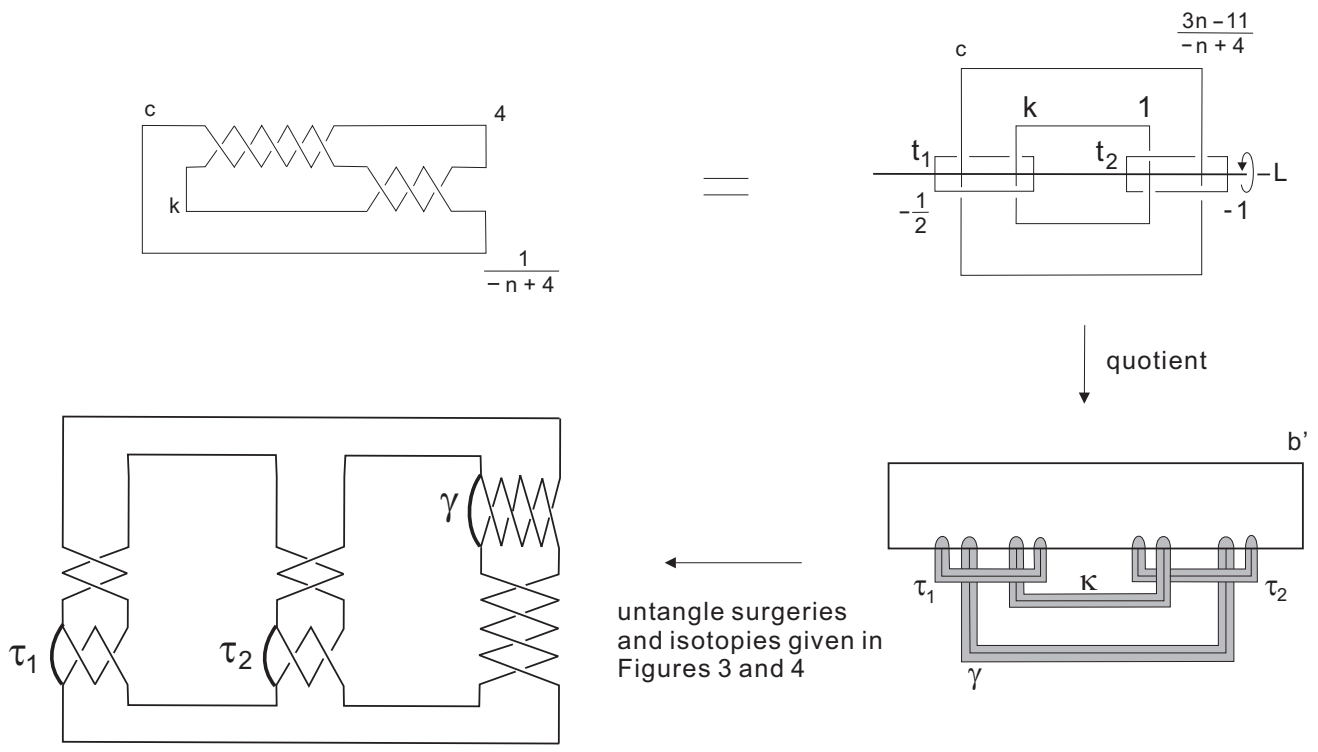

Figure 7: Positions of exceptional fibers

From Figure 7 we recognize that $t_{1}, t_{2}$ and $c$ become exceptional fibers of indices 5,3 and $|4 n-15|$, respectively in $\left(K_{n} ; n\right)$.

For $n \neq 3,4,5, c_{n}$ becomes an exceptional fiber of index $|4 n-15|$, which is the unique maximal index, in $\left(K_{n} ; n\right)$. Experiments via "SnapPea" 31 suggest that $c_{n}$ is a shortest closed geodesic in $S^{3}-K_{n}(n \neq 3,4,5)$. For sufficiently large $|n|$, hyperbolic Dehn surgery theorem [29], 30] shows that $c_{n}$ is the unique shortest closed geodesic in $S^{3}-K_{n}$.

Let us assume that $n=3,4,5$. Then we have put $K_{n}$ as the mirror image of $K_{-n}$ in the proof of Theorem 1.1. Let $k^{\prime} \cup c^{\prime}$ be the mirror image of the link $k \cup c$. Then $K_{n}$ is obtained also from $k^{\prime}$ by $\frac{1}{-n-4}$-surgery on $c^{\prime}$ (i.e., $(n+4)$-twist along $c^{\prime}$ ); we denote the core of the filled solid torus by $c_{n}^{\prime}$. Note that there is an orientation reversing diffeomorphism from $\left(K_{-n} ;-n\right)$ to $\left(K_{n} ; n\right)$ sending $c_{-n}$ (regarded as a fiber in $\left.\left(K_{-n} ;-n\right)\right)$ to $c_{n}^{\prime}$ (regarded as a fiber in $\left(K_{n} ; n\right)$ ). Thus the above observation implies that $c_{n}^{\prime}$ becomes an exceptional fiber of index $|4 n+15|$, which is the unique maximal index, in $\left(K_{n} ; n\right)(n=3,4,5)$. 


\section{References}

[1] M Aït Nouh, D Matignon, K Motegi; Twisted unknots, C. R. Acad. Sci. Paris, Ser. I 337 (2003), 321-326. MathReview

[2] M Aït Nouh, D Matignon, K Motegi; Obtaining graph knots by twisting unknots, Topology Appl. 146-147 (2005), 105-121. MathReview

[3] M Aït Nouh, D Matignon, K Motegi; Geometric types of twisted knots, preprint.

[4] J Berge; Some knots with surgeries yielding lens spaces, unpublished manuscript.

[5] S A Bleiler; Knots prime on many strings, Trans. Amer. Math. Soc. 282 (1984), 385-401. MathReview

[6] S A Bleiler; Prime tangles and composite knots, in: Knot theory and manifolds (Vancouver, B.C. 1983), Lect. Notes in Math. vol. 1144, Springer-Verlag, 1985, pp. 1-13. MathReview

[7] M Culler, C McA Gordon, J Luecke, P B Shalen; Dehn surgery on knots, Ann. Math. 125 (1987), 237-300. MathReview

[8] M Eudave-Muñoz; On hyperbolic knots with Seifert fibered Dehn surgeries, Topology Appl. 121 (2002), 119-141. MathReview

[9] R Fintushel, R J Stern; Constructing lens spaces by surgery on knots, Math. Z. 175 (1980), 33-51. MathReview

[10] D Gabai; Foliations and the topology of 3-manifolds. III, J. Differential Geom. 26 (1987), 479-536. MathReview

[11] F González-Acuña, H Short; Knot surgery and primeness, Math. Proc. Cambridge Philos. Soc. 99 (1986), 89-102. MathReview

[12] C McA Gordon; Dehn Filling; a survey, Proc. Mini Semester in Knot Theory, Banach Center, Warsaw, Poland, 1995.

[13] C McA Gordon, J Luecke; Only integral surgeries can yield reducible manifolds, Math. Proc. Cambridge Philos. Soc. 102 (1987), 97-101. MathReview

[14] C McA Gordon, J Luecke; Knots are determined by their complements, J. Amer. Math. Soc. 2 (1989), 371-415. MathReview

[15] C McA Gordon, J Luecke; Dehn surgeries on knots creating essential tori, I, Comm. Anal. Geom. 4 (1995), 597-644. MathReview

[16] C McA Gordon, J Luecke; Non-integral toroidal Dehn surgeries, Comm. Anal. Geom. 12 (2004), 417-485. MathReview

[17] K Ichihara, K Motegi, H-J Song; Longitudinal Seifert fibered surgeries on hyperbolic knots, preprint.

[18] M Kouno, K Motegi, T Shibuya; Twisting and knot types, J. Math. Soc. Japan 44 (1992), 199-216. MathReview 
[19] P Kronheimer, T Mrowka; Dehn surgery, the fundamental group and SU(2). arXiv:math.GT/0312322

[20] P Kronheimer, T Mrowka, P Ozsváth, Z Szabó; Monopoles and lens space surgeries. arXiv:math.GT/0310164

[21] Y Mathieu, Unknotting, knotting by twists on disks and property (P) for knots in $S^{3}$, Knots 90 (ed. by Kawauchi), Proc. 1990 Osaka Conf. on Knot Theory and Related Topics, de Gruyter, 1992, 93-102 MathReview

[22] T Mattman, K Miyazaki, K Motegi; Seifert fibered surgeries which do not arise from primitive/Seifert-fibered constructions, Trans. Amer. Math. Soc. (to appear).

[23] W Menasco; Closed incompressible surfaces in alternating knot and link complements, Topology 23 (1984), 37-44. MathReview

[24] K Miyazaki, K Motegi; Seifert fibered manifolds and Dehn surgery III, Comm. Anal. Geom. 7 (1999), 551-582. MathReview

[25] J M Montesinos; Surgery on links and double branched coverings of $S^{3}$, in: Knots, groups, and 3-manifolds (Papers dedicated to the memory of R H Fox), Ann. Math. Studies 84 (1975), 227-260. MathReview

[26] K Morimoto; There are knots whose tunnel numbers go down under connected sum, Proc. Amer. Math. Soc. 123 (1995), 3527-3532. MathReview

[27] K Motegi; An experimental study of Seifert fibered Dehn surgery via SnapPea, in: Proceedings of the Winter Workshop of Topology/Workshop of Topology and Computer (Sendai, 2002/Nara, 2001), Interdisc. Inform. Sci. 9 (2003), 95-125. MathReview

[28] M Teragaito; Toroidal surgeries on hyperbolic knots, Proc. Amer. Math. Soc. 130 (2002), 2803-2808. MathReview

[29] W P Thurston; The geometry and topology of 3-manifolds, Lecture notes, Princeton University, 1979. http://www.msri.org/publications/books/gt3m/

[30] W P Thurston; Three dimensional manifolds, Kleinian groups and hyperbolic geometry, Bull. Amer. Math. Soc. 6 (1982), 357-381. MathReview

[31] J Weeks; SnapPea: a computer program for creating and studying hyperbolic 3-manifolds, freely available from http://geometrygames.org/SnapPea/

Department of Mathematics, Nihon University

Tokyo 156-8550, Japan

and

Division of Mathematical Sciences, Pukyong National University

599-1 Daeyondong, Namgu, Pusan 608-737, Korea

Email: motegi@math.chs.nihon-u.ac.jp and hjsong@pknu.ac.kr

Received: 10 March 2005 Revised: 25 March 2005 